\title{
Image De-noising by Various Filters for Different Noise
}

\author{
Pawan Patidar \\ Research Scholar (M. Tech.), Computer \\ Science Department, Poornima College of \\ Engineering, Jaipur, India,
}

\author{
Sumit Srivastava \\ Associate Professor, Computer Science \\ Department, Poornima College of Engineering, \\ Jaipur (Rajasthan), India
}

\begin{abstract}
Image processing is basically the use of computer algorithms to perform image processing on digital images. Digital image processing is a part of digital signal processing. Digital image processing has many significant advantages over analog image processing. Image processing allows a much wider range of algorithms to be applied to the input data and can avoid problems such as the build-up of noise and signal distortion during processing of images. Wavelet transforms have become a very powerful tool for de-noising an image. One of the most popular methods is wiener filter. In this work four types of noise (Gaussian noise, Salt \& Pepper noise, Speckle noise and Poisson noise) is used and image de-noising performed for different noise by Mean filter, Median filter and Wiener filter . Further results have been compared for all noises.
\end{abstract}

\section{Keywords}

Wavelet Transform, Gaussian noise, Salt \& Pepper noise, Speckle noise, Poisson noise, Wiener Filter.

\section{INTRODUCTION}

Image de-noising is an vital image processing task i.e. as a process itself as well as a component in other processes. There are many ways to de-noise an image or a set of data and methods exists. The important property of a good image denoising model is that it should completely remove noise as far as possible as well as preserve edges. Traditionally, there are two types of models i.e. linear model and non-liner model. Generally, linear models are used. The benefits of linear noise removing models is the speed and the limitations of the linear models is, the models are not able to preserve edges of the images in a efficient manner i.e the edges, which are recognized as discontinuities in the image, are smeared out. On the other hand, Non-linear models can handle edges in a much better way than linear models. One popular model for nonlinear image denoising is the Total Variation (TV)-filter.

We suggest to de-noise a degraded image $\mathrm{X}$ given by $\mathrm{X}=\mathrm{S}+\mathrm{N}$, where $\mathrm{S}$ is the original image and $\mathrm{N}$ is an Additive White Gaussian noise with unknown variance [2]. The rest of the paper is organized as follows:-

- In the second section we review the wavelet transform.

- In the third section we present the method of Average filter.

- In the fourth section we present the method of Median filter.
- In the fifth section we present the method of Wiener filter.

- In the sixth section we described types of noise.

- The simulation results are discussed in part seven.

- We conclude and future work in part eight and nine.

\section{WAVELET TRANSFORM}

In several applications, it might be essential to analyze a given signal. The structure and features of the given signal may be better understood by transforming the data into another domain. There are several transforms available like the Fourier transform, Hilbert transform, wavelet transform, etc. The Fourier transform is probably the most popular transform. However the Fourier transform gives only the frequencyamplitude representation of the raw signal. The time information is lost. So we cannot use theFourier transform in applications which require both time as well as frequency information at the same time. The Short Time Fourier Transform (STFT) was developed to overcome this drawback [2].

\section{MEAN FILTER}

We can use linear filtering to remove certain types of noise. Certain filters, such as averaging or Gaussian filters, are appropriate for this purpose. For example, an averaging filter is useful for removing grain noise from a photograph. Because each pixel gets set to the average of the pixels in its neighborhood, local variations caused by grain are reduced. Conventionally linear filtering Algorithms were applied for image processing. The fundamental and the simplest of these algorithms is the Mean Filter as defined in [6].The Mean Filter is a linear filter which uses a mask over each pixel in the signal. Each of the components of the pixels which fall under the mask are averaged together to form a single pixel. This filter is also called as average filter. The Mean Filter is poor in edge preserving. The Mean filter is defined by:

$$
\begin{gathered}
1 \mathrm{~N} \\
\text { Mean filter }\left(\mathrm{x}_{1} \ldots . . \mathrm{x}_{\mathrm{N}}\right)=-\sum_{\mathrm{N}=1} \mathrm{x}_{\mathrm{i}}
\end{gathered}
$$

where $\left(x_{1} \ldots . x_{N}\right)$ is the image pixel range. Generally linear filters are used for noise suppression.

\section{MEDIAN FILTER}

The Median filter is a nonlinear digital filtering technique, often used to remove noise. Such noise reduction is a typical preprocessing step to improve the results of later processing (for example, edge detection on an image). Median filtering is very widely used in digital image processing because under certain 
conditions, it preserves edges whilst removing noise. The main idea of the median filter is to run through the signal entry by entry, replacing each entry with the median of neighboring entries. Note that if the window has an odd number of entries, then the median is simple to define: it is just the middle value after all the entries in the window are sorted numerically. For an even number of entries, there is more than one possible median. The median filter is a robust filter. Median filters are widely used as smoothers for image processing, as well as in signal processing and time series processing. A major advantage of the median filter over linear filters is that the median filter can eliminate the effect of input noise values with extremely large magnitudes. (In contrast, linear filters are sensitive to this type of noise - that is, the output may be degraded severely by even by a small fraction of anomalous noise values) [6]. The output $y$ of the median filter at the moment $\mathrm{t}$ is calculated as the median of the input values corresponding to the moments adjacent to t:

$y(t)=\operatorname{median}\left(\left(x(t-T / 2), x\left(t-T_{1}+1\right), \ldots, x(t), \ldots, x(t+T / 2)\right)\right.$

where $t$ is the size of the window of the median filter. Besides the one-dimensional median filter described above, there are two-dimensional filters used in image processing .Normally images are represented in discrete form as twodimensional arrays of image elements, or "pixels" - i.e. sets of non-negative values $B_{i j}$ ordered by two indexes -

$$
\mathrm{i}=1, \ldots, \mathrm{N}_{\mathrm{y}} \text { (rows) and } \mathrm{j}=1, \ldots, \mathrm{N}_{\mathrm{y}}(\text { column }) \text {. }
$$

where the elements $B_{i j}$ are scalar values, there are methods for processing color images, where each pixel is represented by several values, e.g. by its "red", "green", "blue" values determining the color of the pixel.

\section{WIENER FILTER}

The goal of the Wiener filter is to filter out noise that has corrupted a signal. It is based on a statistical approach. Typical filters are designed for a desired frequency response. The Wiener filter approaches filtering from a different angle. One is assumed to have knowledge of the spectral properties of the original signal and the noise, and one seeks the LTI filter whose output would come as close to the original signal as possible [1]. Wiener filters are characterized by the following:

\footnotetext{
a. Assumption: signal and (additive) noise are stationary linear random processes with known spectral characteristics.

b. Requirement: the filter must be physically realizable, i.e. causal (this requirement can be dropped, resulting in a non-causal solution)

c. Performance criteria: minimum mean-square error
}

\subsection{Wiener Filter in the Fourier Domain}

The Wiener filter is:

$$
G(u, v)=\frac{H^{*}(u, v) P_{s}(u, v)}{|H(u, v)|^{2} P_{s}(u, v)+P_{n}(u, v)}
$$

Dividing through by $\mathbf{P}_{\mathrm{s}}$ makes its behavior easier to explain:

$$
G(u, v)=\frac{H^{*}(u, v)}{|H(u, v)|^{2}+\frac{P_{n}(u, v)}{P_{s}(u, v)}}
$$

where

$\mathrm{H}(\mathrm{u}, \mathrm{v})=$ Degradation function

$\mathrm{H}^{*}(\mathrm{u}, \mathrm{v})=$ Complex conjugate of degradation function

Pn $(\mathrm{u}, \mathrm{v})=$ Power Spectral Density of Noise

Ps $(u, v)=$ Power Spectral Density of un-degraded image

The term $\mathbf{P}_{\mathbf{n}} / \mathbf{P}_{\mathbf{s}}$ can be interpreted as the reciprocal of the signalto-noise ratio.

\section{IMAGE NOISE}

Image noise is the random variation of brightness or color information in images produced by the sensor and circuitry of a scanner or digital camera. Image noise can also originate in film grain and in the unavoidable shot noise of an ideal photon detector [4].Image noise is generally regarded as an undesirable by-product of image capture. Although these unwanted fluctuations became known as "noise" by analogy with unwanted sound they are inaudible and such as dithering. The types of Noise are following:-

- Amplifier noise (Gaussian noise)

- $\quad$ Salt-and-pepper noise

- Shot noise(Poisson noise)

- $\quad$ Speckle noise

\subsection{Amplifier noise (Gaussian noise)}

The standard model of amplifier noise is additive, Gaussian, independent at each pixel and independent of the signal intensity.In color cameras where more amplification is used in the blue color channel than in the green or red channel, there can be more noise in the blue channel .Amplifier noise is a major part of the "read noise" of an image sensor, that is, of the constant noise level in dark areas of the image [4].

\subsection{Salt-and-pepper noise}

An image containing salt-and-pepper noise will have dark pixels in bright regions and bright pixels in dark regions [4]. This type of noise can be caused by dead pixels, analog-to-digital converter errors, bit errors in transmission, etc.This can be eliminated in large part by using dark frame subtraction and by interpolating around dark/bright pixels.

\subsection{Poisson noise}

Poisson noise or shot noise is a type of electronic noise that occurs when the finite number of particles that carry energy, such as electrons in an electronic circuit or photons in an optical device, is small enough to give rise to detectable statistical fluctuations in a measurement [4]. 


\subsection{Speckle noise}

Speckle noise is a granular noise that inherently exists in and degrades the quality of the active radar and synthetic aperture radar (SAR) images. Speckle noise in conventional radar results from random fluctuations in the return signal from an object that is no bigger than a single image-processing element. It increases the mean grey level of a local area. Speckle noise in SAR is generally more serious, causing difficulties for image interpretation. It is caused by coherent processing of backscattered signals from multiple distributed targets. In SAR oceanography [5], for example, speckle noise is caused by signals from elementary scatters, the gravity-capillary ripples, and manifests as a pedestal image, beneath the image of the sea waves.

\section{SIMULATION RESULTS}

The Original Image is Nayantara image, adding four types of Noise (Gaussian noise, Poisson noise, Speckle noise and Salt \& Pepper noise).adding the noise with standard deviation(0.025) and De-noised image using Mean filter, Median filter and Wiener filter and comparisons among them.

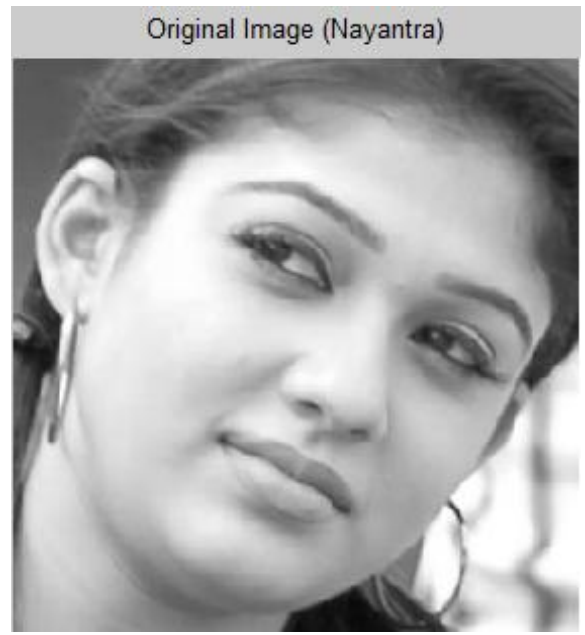

Fig.1 Nayantara Image

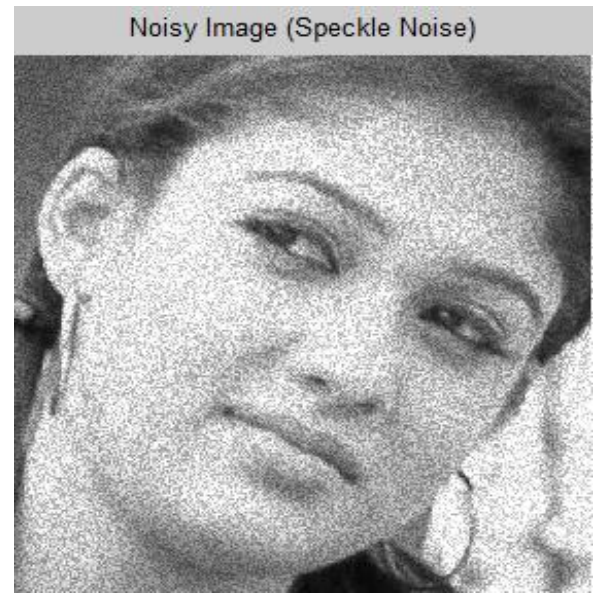

Figure 2: adding speckle noise with standard deviation (0.025)

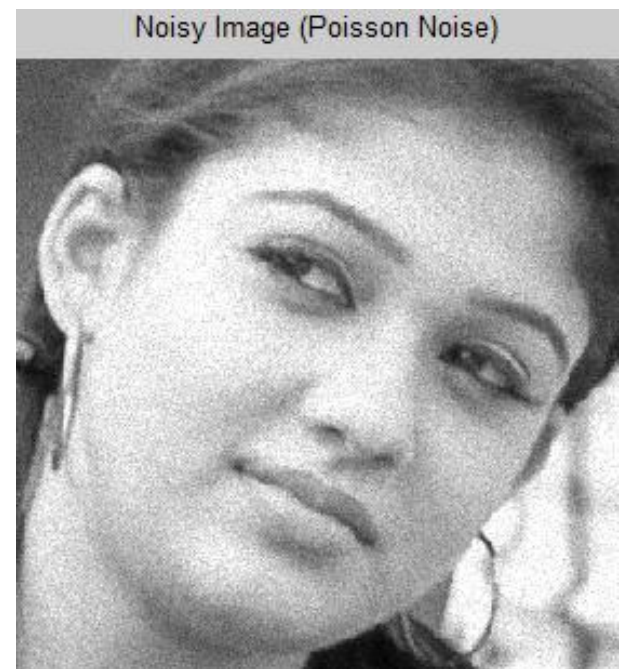

Figure 3: adding Poisson noise with standard deviation (0.025)

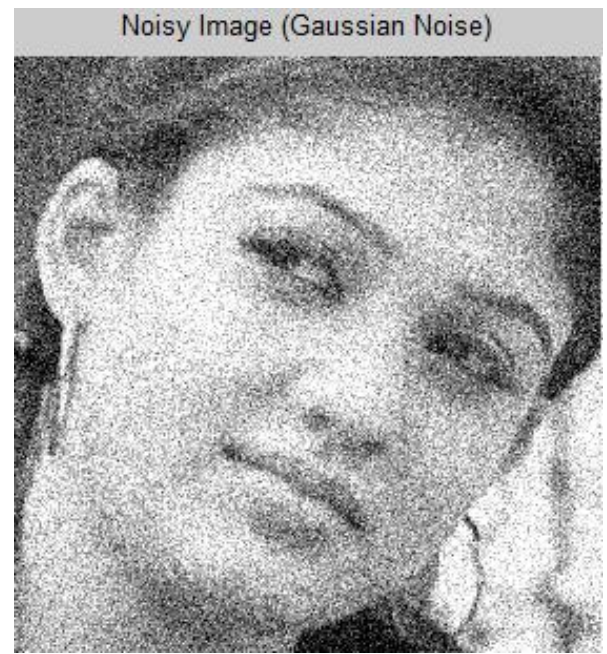

Figure 4: adding Gaussian noise with standard deviation (0.025)

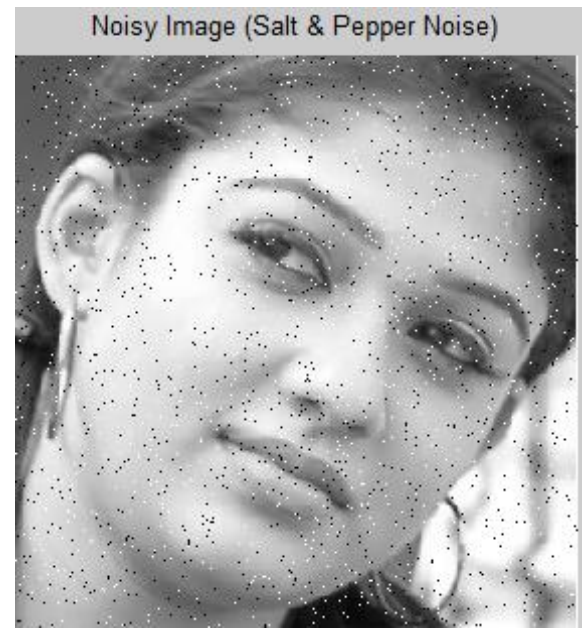

Figure 5: adding salt\& pepper noise with standard deviation (0.025) 
De-noising by Average filter(for speckle noise)

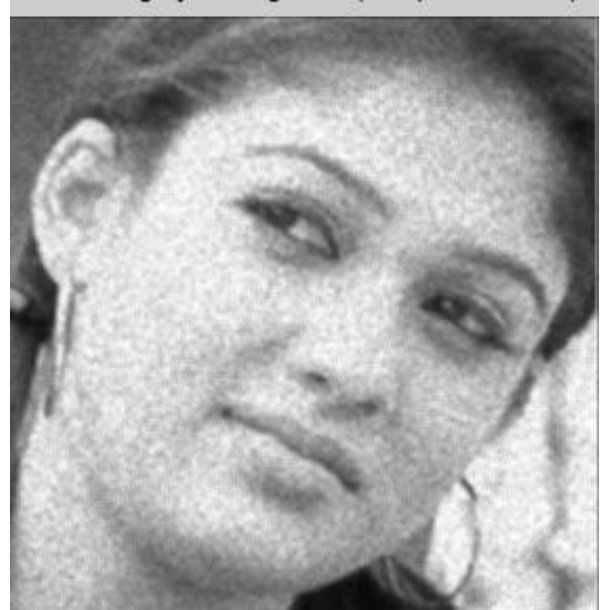

Figure 6:De-noising by mean filter

De-noising by Average filter(for poisson noise)

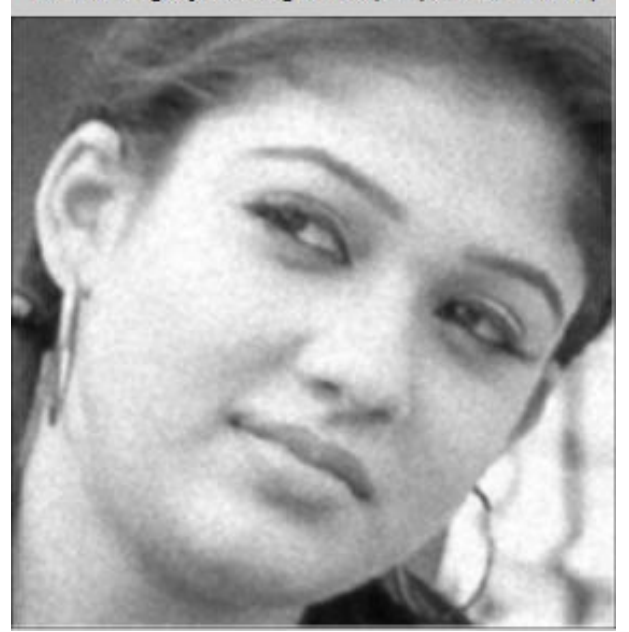

Figure 7: De-noising by mean filter

De-noising by Average filter(for gaussian noise)

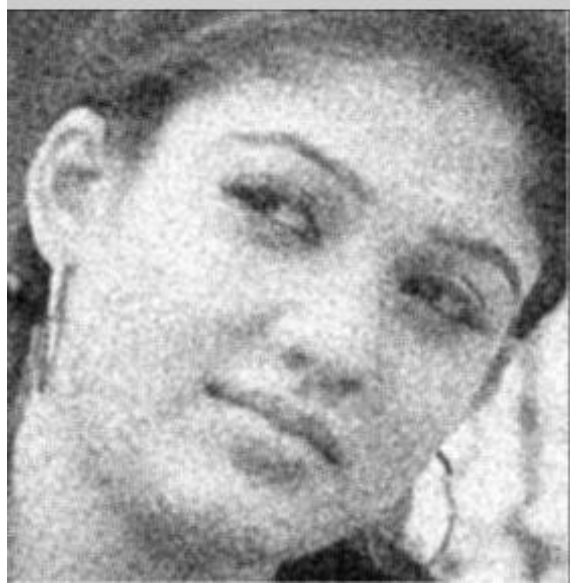

Figure 8: De-noising by mean filter

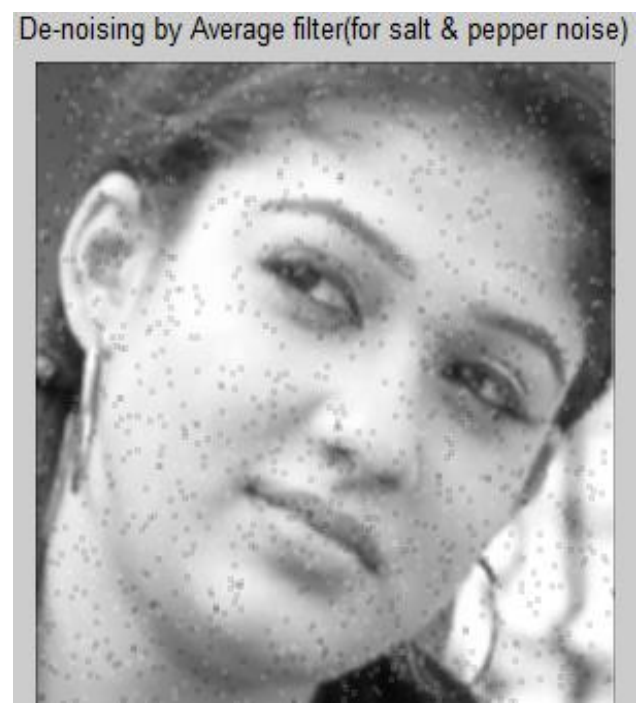

Figure 9: De-noising by mean filter

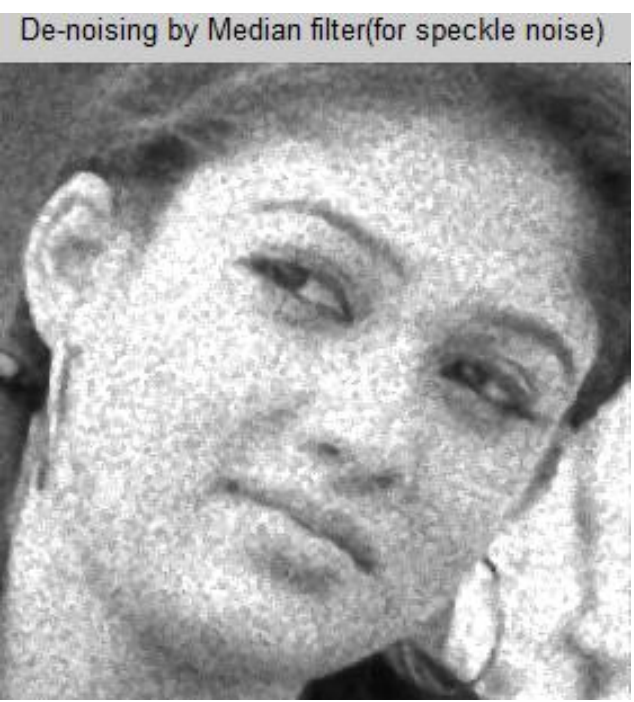

Figure 10: De-noising by median filter

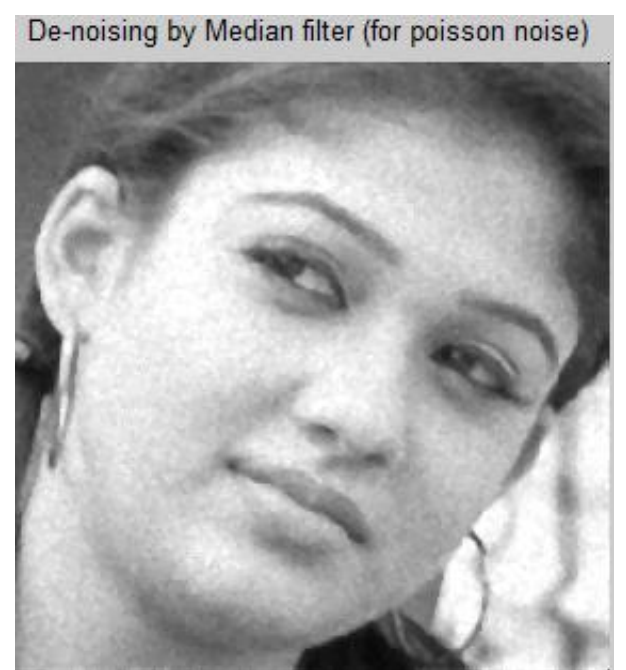

Figure 11: De-noising by median filter 

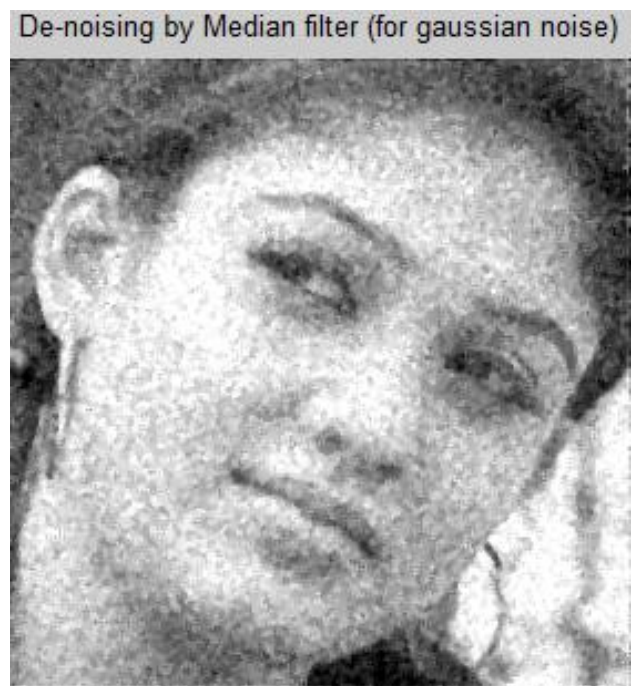

Figure 12: De-noising by median filter

De-noising by Median filter (for salt \& pepper noise)

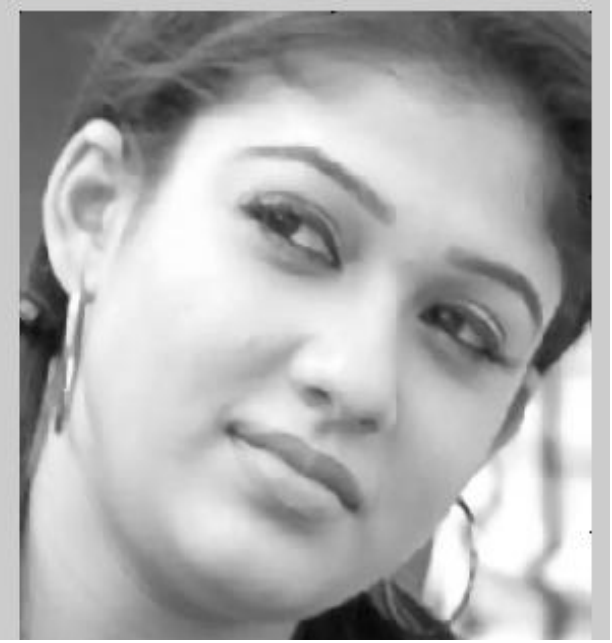

Figure 13: De-noising by median filter

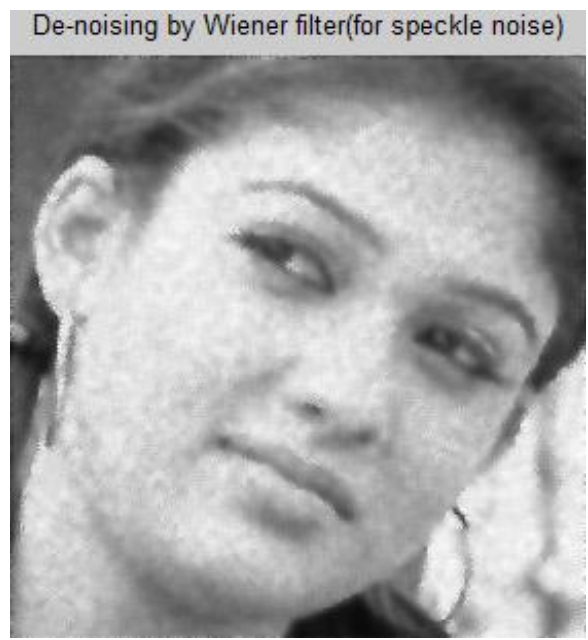

Figure 14: De-noising by wiener filter

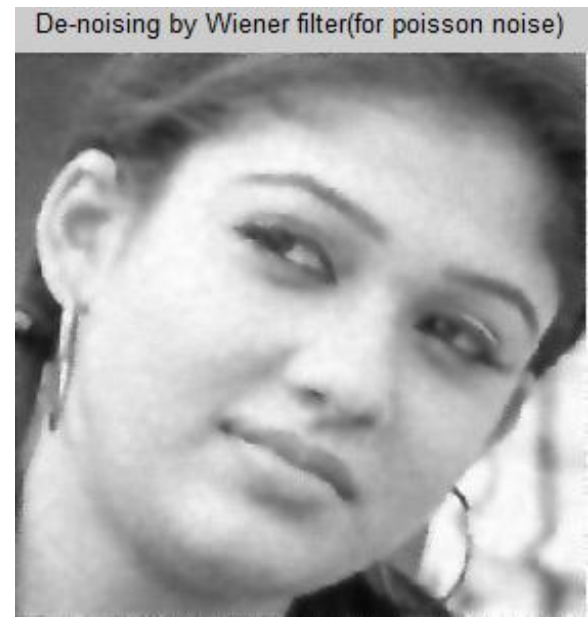

Figure 15: De-noising by median filter

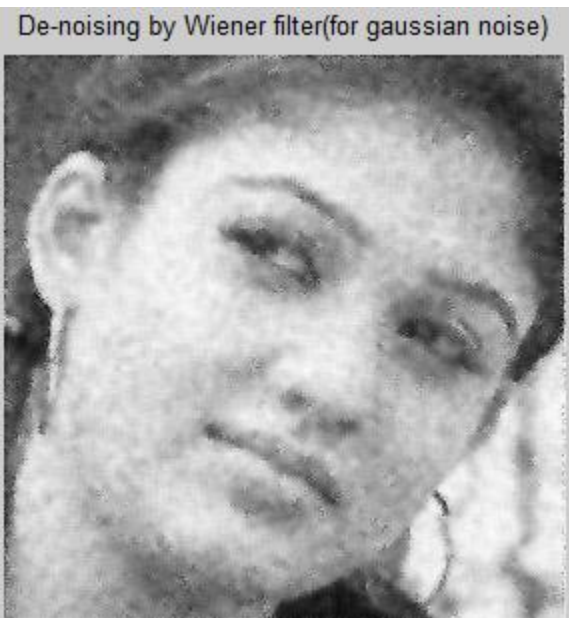

Figure 16: De-noising by median filter

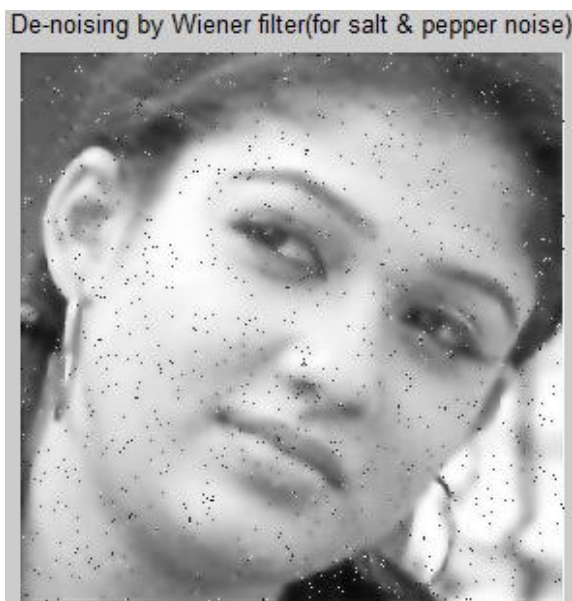

Figure 17: De-noising by median filter

\section{CONCLUSION}

We used the Nayantara Image(figure 1) in "png" format ,adding four noise (Speckle, Gaussian ,Poisson and Salt \& Pepper) in original image with standard deviation(0.025) (figure 2 to figure 
$5)$,De-noised all noisy images by all filters and conclude from the results (figure 6 to figure 17) that:

(a)The performance of the Wiener Filter after de-noising for all Speckle, Poisson and Gaussian noise is better than Mean filter and Median filter.

(b)The performance of the Median filter after de-noising for all Salt \& Pepper noise is better than Mean filter and Wiener filter.

\section{SCOPE FOR FUTURE WORK}

There are a couple of areas which we would like to improve on. One area is in improving the de-noising along the edges as the method we used did not perform so well along the edges. Another area of improvement would be to develop a better optimality criterion as the MSE is not always the best optimality criterion. The future work of research would be to implement Wiener Filter in Wavelet Domain, applying the methods in which the noise variance is known \& in which the noise variance is unknown i.e. the MAD method.

\section{REFERENCES}

[1] Wavelet domain image de-noising by thresholding and Wiener filtering.Kazubek, M. Signal Processing Letters, IEEE, Volume: 10, Issue: 11, Nov. 2003265 Vol.3.

[2] Wavelet Shrinkage and W.V.D.: A 10-minute Tour Donoho, D.L; (David L. Donoho's website).

[3] Image De-noising using Wavelet Thresholding and Model Selection.Shi Zhong Image Processing, 2000, Proceedings, 2000 International Conference on, Volume: 3, 10-13 Sept. 2000 Pages: 262.

[4] Charles Boncelet (2005).’'Image Noise Models". in Alan C. Bovik. Handbook of Image and Video Processing.

[5] Sedef Kent, Osman Nuri Oçan, and Tolga Ensari (2004). "Speckle Reduction of Synthetic Aperture Radar Images Using Wavelet Filtering". in astrium. EUSAR 2004 Proceedings, 5th European Conference on Synthetic Aperture Radar, May 25-27, 2004, Ulm, Germany.

[6] James C. Church, Yixin Chen, and Stephen V. Rice Department of Computer and Information Science, University of Mississippi, "A Spatial Median Filter for Noise Removal in Digital Images", IEEE, page(s): 618623, 2008. 